\title{
SIMULATION OF CONJUGATE CONVECTIVE-CONDUCTIVE HEAT TRANSFER IN A MICROCHANNEL WITHIN THE SLIP REGIME USING GPU ACCELERATED LATTICE BOLTZMANN
}

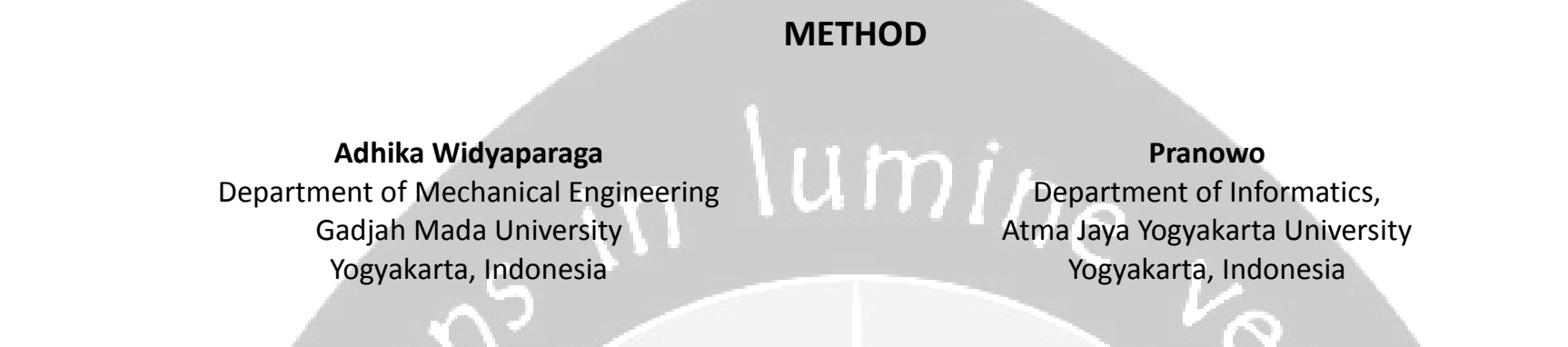

\section{ABSTRACT}

This study aims to investigate the significance of conjugate heat transfer in the microscale within the slip regime. As within the slip regime the continuum assumption is invalid due to presence of rarefaction effects, the Lattice Boltzmann method (LBM) is employed to overcome the limitations of Navier Stokes based solutions in this regime. We have constructed and compared two case models in which a fluid of higher temperature enters a microchannel. The conditions are set to obtain Knudsen numbers which result in the slip regime being dominant. To investigate the effect of conjugate heat transfer, the two models differed in the aspect that one model did not incorporate conjugate heat transfer and while the other did. The numerical calculation was validated by comparing the velocity profile results to exact theoretical approximations and was found to agree well. The results of comparison of models Case I and Case 2 have shown that temperature profile is affected significantly by conjugate heat transfer. The conjugate heat transfer at the microchannel wall (Case 1) was shown to maintain the initial temperature of fluid longer than compared to a purely isothermal wall (Case 2), thus signifying the importance of the consideration of conjugate heat transfer effects in microfluid models. We have implemented GPU based parallel processing to reduce computation time. The result of the incorporation of GPU processing was found to increase processing speed up to 15 times.

\section{INTRODUCTION}

Recently research in the field of fluid flow and heat transfer in microchannel has been attracted many researchers, due to the rapid growth of the advanced microdevices such as MEMS. It is important to understand the phenomenons that occur in microchannels for designing these microdevices. Unfortunately, as stated by Kandiklar[1] that the construction of micro devices has been conducted without a complete understanding of the way physical processes may have been altered by the miniaturized geometry. One important method to understand processes at the microscale is numerical modeling, since experimental measurements are often difficult to conduct at the microscale.

Numerical method modeling is powerful tool in investigating microscale phenomena and clarifying the predominant factors that influenced the phenomena.

Extensive numerical research works have been done in this field. Most of them are based on traditional numerical methods such as finite difference, finite volume and finite element for solving the Navier Stokes Equations as the governing equations. Sung and Mudawar[2] used experimental and numerical methods to explore the cooling performance of device consisting of a jet impingement/microchannel at high Reynolds number. They showed that heat transfer characteristic in small flow passage at high Reynolds number can be predicted accurately by using the Navier Stokes equations and turbulence model. It should be noted that they did not include the effect of the low Reynolds number. Li et al[3] conducted a study to reveal the flow and heat transfer in microtubes at the Reynolds number from 20 to 2400 by using experimental and numerical methods. They used Navier Stokes equations as the governing equations and the finite volume method for discretization. The experimental results showed that the Nusselt number do not accord with the conventional results when the Reynolds number is low and relative thickness of the tube wall is high. The numerical study showed the tube wall thickness has significant effect for low Reynolds.

The Navier-Stokes and energy equations were based on the assumption that the fluid can be treated as continuum. This assumption may break down in some fluid flow regimes, such as fluid flow in microchannel at low Reynolds number, where rarefaction effects are significant. Therefore, a different modeling approach should be conducted. The Lattice 
Boltzmann method (LBM) is one such method that can be used for fluid flow and heat transfer modeling in fluid flow regimes ranging from mesoscale (slip flow regime) to macroscale since LBM is based on Boltzmann gas kinetic theory. Shu et al[4] proposed a lattice Boltzmann BGK model for simulation of micro flows with heat transfer. They used a diffuse scattering boundary condition (DSBC) considering the velocity slip and temperature jump at wall boundaries. The comparisons with direct simulation Monte Carlo (DSMC) and molecular dynamics (MD) show good agreement. Ansumali et al[5] proposed a new computational approach for the simulation of micro flows called entropic lattice Boltzmann method. They proved that their proposed method predicted fluid flow characteristic accurately for various regimes (from the continuous case to the free-molecular flow). Celik[6] studied fluid flow and heat transfer in two-dimensional microchannels numerically using LBM. Slip velocity and temperature jump boundary conditions are used for the micro channel simulations. The velocity profiles and Nusselt numbers are compared to analytical results and a good match was observed. To accelerate computation time, GPU parallel programming written in Matlab and Jacket software was used and a processing speedup of 14 times was achieved.

Most of microchannel researches neglected the effect of conjugate heat conduction through the solid microchannel wall. The boundary condition can be simplified as constant wall temperature (CWT) or constant wall heat flux (CWHF) at the fluid-solid interfaces. However, the flow patterns and heat transfer cofficients might be affected strongly by the presence of heat conduction in the solid wall. Therefore, conjugate heat transfer effects should be considered in numerical modeling[7]. Pirouz[8]investigated conjugate heat transfer in rectangular channel using LBM with Reynolds numbers ranging to 200 to 1000 and various thermal diffusivities. They show that LBM is suitable for the study of heat transfer in conjugate problems. Wang et al.[9] developed a lattice boltzmann algorithm for fluid-solid conjugate heat transfer. The developed scheme agrees well with the classical CFD for predicting of microchannel flow. However, both works above did not consider a slip boundary condition in their calculations.

In this paper, we propose the implementation of parallel LBM for the simulation of two-dimensional conjugate heat transfer for fluid flow within the slip regime in a microchannel. We propose the use of graphical processing unit (GPU) parallel processing to accelerate the computational process. The LBM code was implemented by using NVIDIA C language and was run on a Nvidia GeForce 635M graphics processor.

\section{LATTICE BOLTZMANN METHOD}

The Lattice Boltzmann Method originated from the lattice gas automata[10]. It is based on the discretization of the
Boltzmann Equation with the Bhatnagar-Gross-Krook (BGK) relaxation for the collision operator.

Two kinds of particle distribution functions are used for for modeling the natural convective flows, i.e. density distribution functions for calculation of density and velocity fields and energy distribution functions for calculation of the temperature[9] :

$$
f_{i}\left(\mathbf{x}+e_{i} \Delta \mathbf{x}, t+e_{i} \Delta t\right)-f_{i}(\mathbf{x}, t)=-\frac{1}{\tau_{f}}\left(f_{i}(\mathbf{x}, t)-f_{i}^{e q}(\mathbf{x}, t)\right)+\mathbf{F}
$$

$$
g_{i}\left(\mathbf{x}+e_{i} \Delta \mathbf{x}, t+e_{i} \Delta t\right)-g_{i}(\mathbf{x}, t)=-\frac{1}{\tau_{f}}\left(g_{i}(\mathbf{x}, t)-g_{i}^{e q}(\mathbf{x}, t)\right)+\mathbf{F}
$$

where $F$ describes external body forces. In our model, no external forces are present and therefore we set $\mathrm{F}$ to be zero. $\tau_{f}$ and $\tau_{g}$ are the relaxation time for momentum and energy transport calculated by :

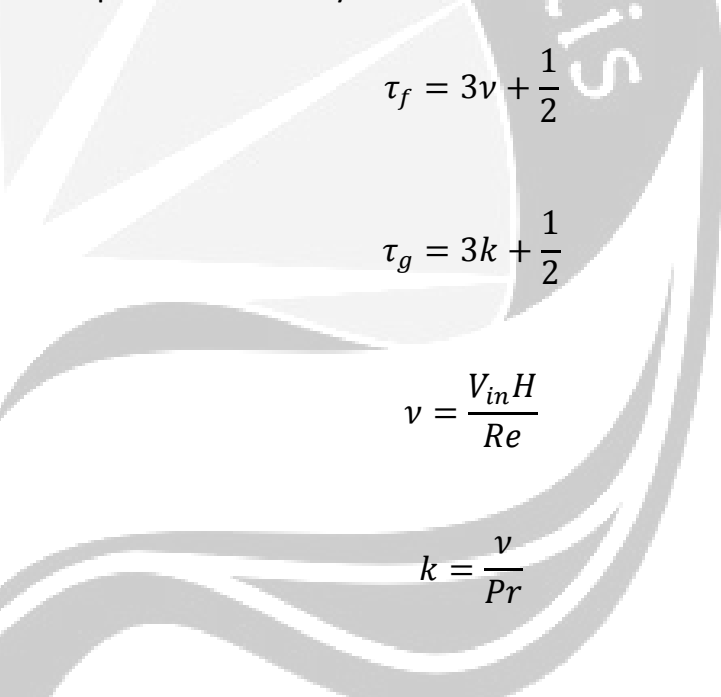

$v$ is the kinematic viscosity, $k$ is thermal diffusivity of fluid, $V_{\text {in }}$ is the velocity of the fluid at the inlet, $\mathrm{Pr}$ is the Prandtl number and $\mathrm{Re}$ is the Reynolds number.

The discretized equilibrium distribution functions are given by:

$$
\begin{gathered}
f_{i}^{e q}=\rho \omega_{n s, i}\left(1+3 e_{i} \cdot \mathbf{u}+4.5\left(e_{i} \cdot \mathbf{u}\right)^{2}-1.5|\mathbf{u}|^{2}\right) \\
g_{i}^{e q}=T \omega_{T, i}\left(1+3 e_{i} \cdot \mathbf{u}\right)
\end{gathered}
$$

Where $\omega_{\mathrm{ns}, \mathrm{i}}$ and $\omega_{\mathrm{T}, \mathrm{i}}$ are the weighting factors for momentum and energy respectively. $u$ is the fluid velocity and $e$ is the discrete velocity. 


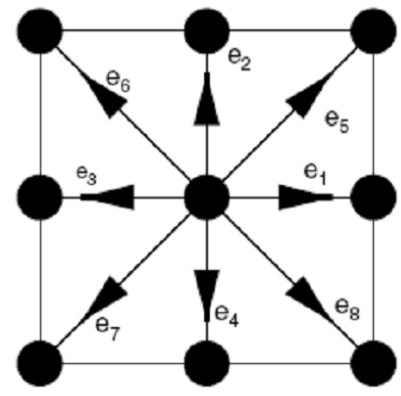

FIGURE 1. DISCRETE VELOCITY FOR D2Q9

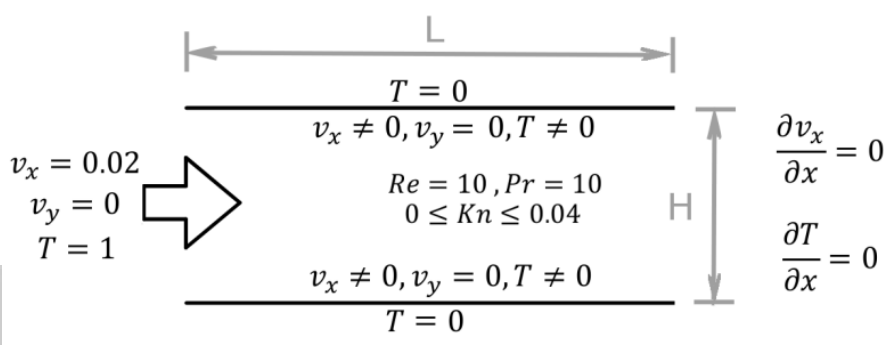

FIGURE 2. GEOMETRY AND BOUNDARY CONDITIONS OF THE FLUID FLOW AND HEAT TRANSFER MODEL WITHOUT CONJUGATE HEAT TRANSFER[1]

\section{CASE 2. WITH CONJUGATE HEAT TRANSFER IN MICROCHANNEL} WALL

In this case, the effect of the conduction within the solid microchannel wall is taken into account. The physical domains are described by Figure 3. The boundary conditions are similar to the first case except the boundary condition at the fluidsolid interface. For this case, we have removed the temperature slip at the wall. The slip velocity is applied at the interface between the wall and the fluid but the velocity components are set to be zero within the solid wall.

The temperature calculation can be easily handled by assigning different thermal diffusivity for the solid. By using this way the flux continuity at the interface will be ensured automatically.

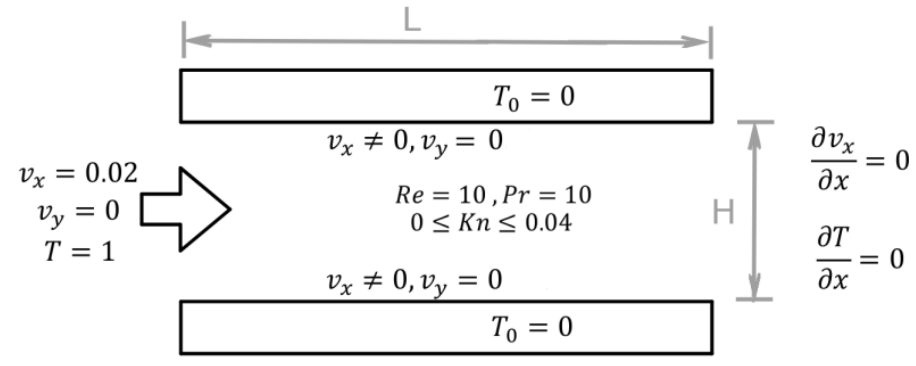

\section{FLUID FLOW AND HEAT TRANSFER IN MICROCHANNEL}

\section{CASE 1. WITHOUT CONJUGATE HEAT TRANSFER}

The case for conditions without conjugate heat transfer was adopted from Celik[6]with the physical domains described by Figure 2. The slip boundary condition and temperature jump are implemented at the fluid-solid interfaces of the channel adopting the formulation by Tian et al.[11]. The velocity profile and the temperature at the inlet are given as shown in Figure 1. The value of velocities and temperature at the outlet are extrapolated from the adjacent lattices. 
same instruction[12]. In relation to the Lattice Boltzmann Method, each nodes can be addressed into block thread and takes into account the value of the offset input.

Basically the algorithm of LBM consists two steps, namely, streaming and colliding. Tölke[13] implemented the LBM using NVIDIA CUDA where he exploited the CUDA shared memory to accelerate the execution time. Accessing shared memory is much faster than accessing global memory. Unfortunately, the capacity of the shared memory is very limited and it needs synchronization. Without synchronization, a race condition will be created and the correctness of the execution results will depend on the nondeterministic details of the hardware [14]. In this paper, despite using shared memory, we used texture memory. The discretization of LBM contained high spatial locality, so the use of texture memory is very suitable. As stated by Sanders and Kandrott [14], texture memory is cached on chip, so it will provide higher effective bandwidth by reducing memory requests to off-chip DRAM. Specifically, texture caches are designed for graphics applications where memory access patterns exhibit a great deal of spatial locality.

The excerpt of the CUDA code that shows the parallel momentum streaming step is given below:

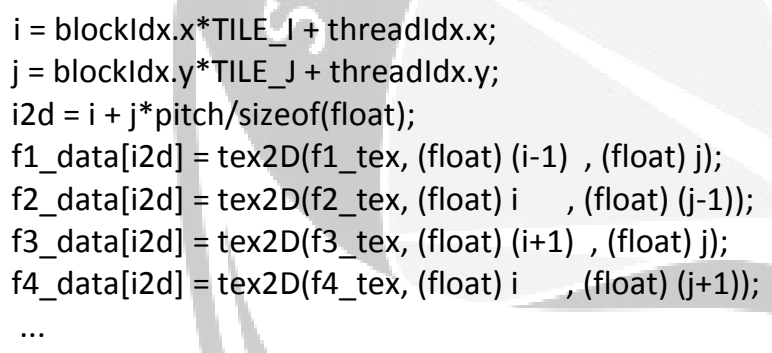

The implementation of the parallel of the momentum colliding step is:

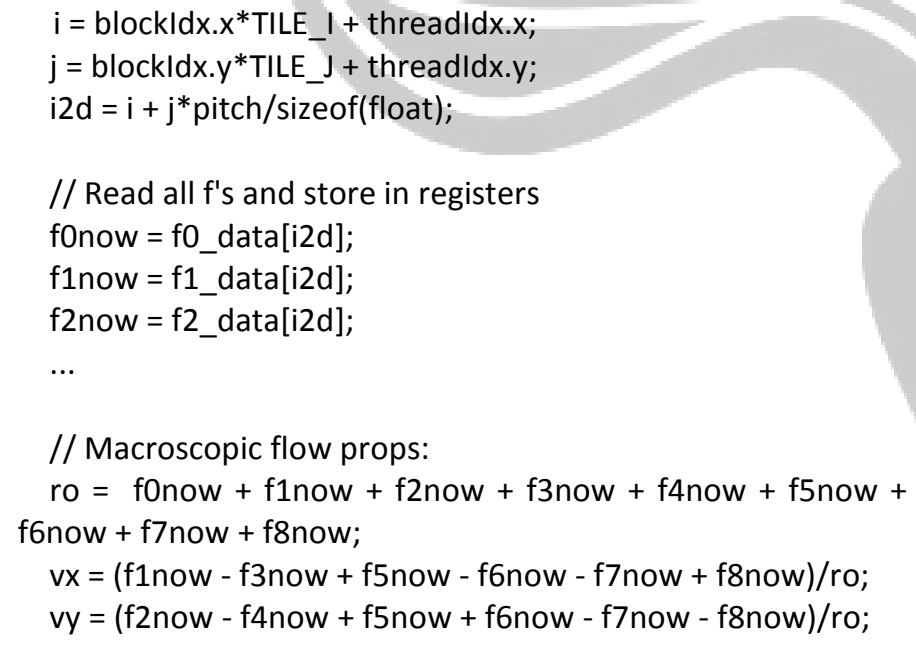

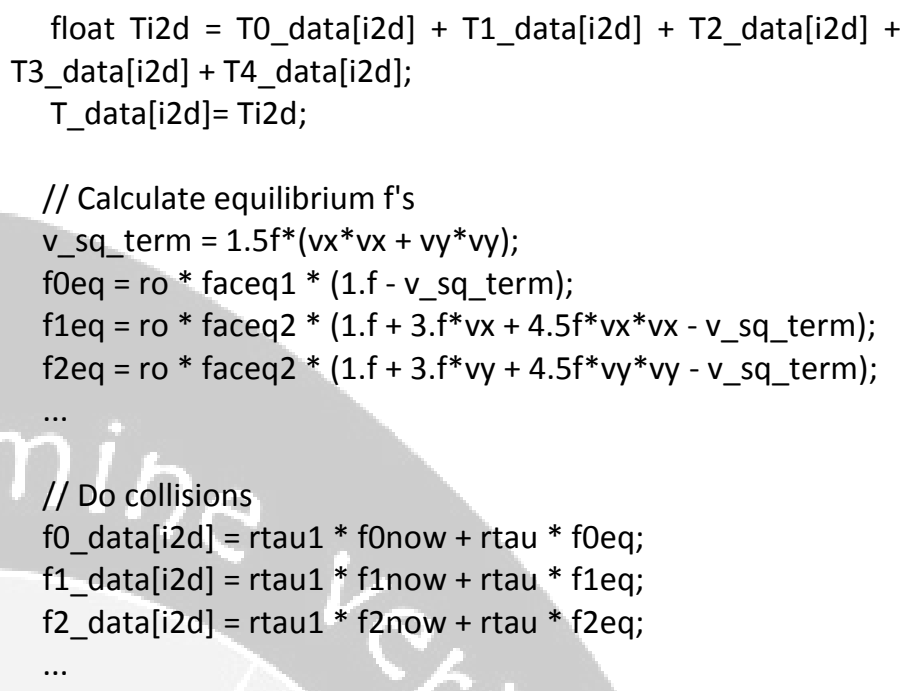

\section{RESULTS AND DISCUSSIONS}

The LBM model was based codes developed by Pullan for hydrodynamical simulation[15]. We have implemented energy distribution functions and the handling of the slip velocity and temperature boundary conditions. The implementation of numerical calculation was conducted on Intel 15 processors and NVIDIA GEforce 635 M GPUs. Here we will discuss the results of the model.

\section{CASE 1. WITHOUT CONJUGATE HEAT TRANSFER}

In this case, the Knudsen number $(\mathrm{Kn})$ numbers were varied from 0 to 0.04 and a constant Reynolds number $\operatorname{Re}=10$ was applied. We used 3 types of grids, consisting of $16 \times 384$, $48 \times 768$, and $80 \times 1536$ nodes. The calculation results of the isotherms at steady states for grid size $48 \times 768$ are shown in Figure 4 . The steady state velocity and temperature profiles near the inlet are shown in Figure 5 and 6 . As can be seen, the evolution of velocity and thermal profile to become hydrodynamically and thermally fully developed flows can be clearly observed, as are the effects of velocity slip and temperature jump. As validation, we have compared the results of the LBM calculation with the exact solution for slip boundary conditions given by Karniadakis et al[16] given in Equation 12. Figure 7 shows that the LBM calculation and the exact solution results for fully developed flow at $\mathrm{x} / \mathrm{H}=2.13$ show excellent agreement. 
FIGURE 4. STEADY STATE ISOTHERM IN CASE 1 MICROCHANNEL FOR GRID SIZE 48X768

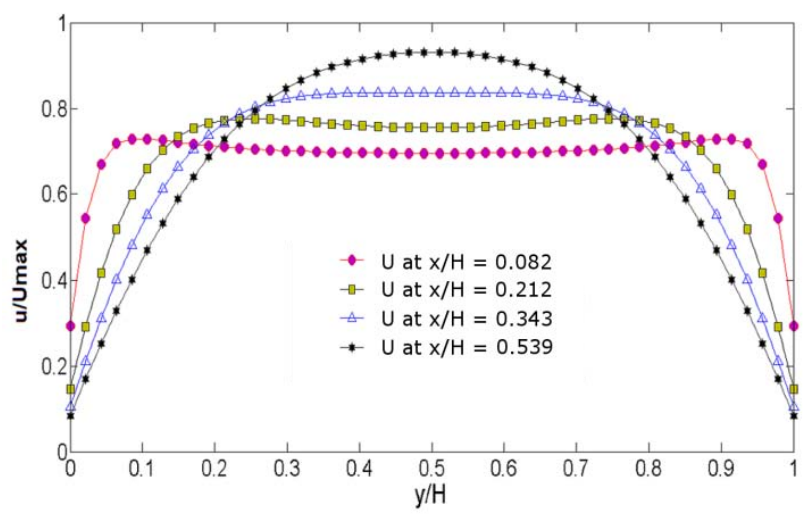

FIGURE 5. STEADY STATE VELOCITY PROFILES NEAR INLET IN MICROCHANNEL FOR CASE I

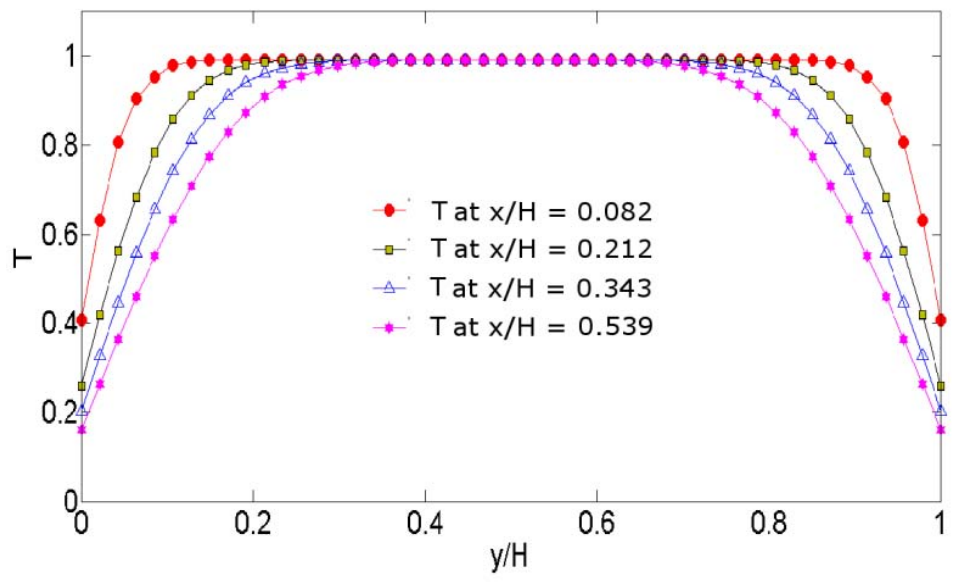

FIGURE 6. STEADY STATE TEMPERATURE PROFILES NEAR INLET IN MICROCHANNEL FOR CASE I

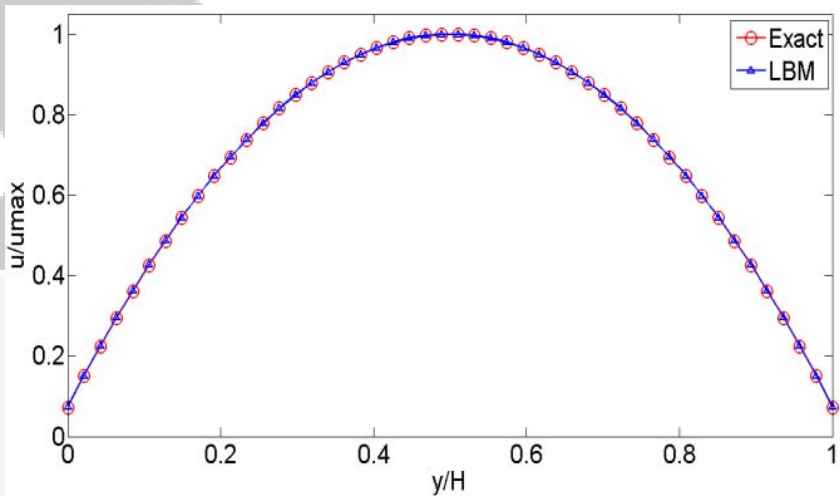

FIGURE 7. COMPARISON OF FULLY DEVELOPED VELOCITY PROFILES OF CASE 1 MODEL AND EXACT SOLUTION AT $X / H=2.13$

$$
u(y)=\frac{-\left(\frac{y}{H}\right)^{2}+\left(\frac{y}{H}\right)+\frac{K n}{(1+K n)}}{\frac{1}{6}+\frac{K n}{(1+K n)}}
$$

Grid independence of the present numerical results has been established for various Knudsen numbers, i.e. 0, 0.02 and 0.04 . Table 1 gives the Nusselt numbers on three grids and compares the current study with calculations by Celik [6]. As can be seen, results show a good agreement.

Table 1. Grid independence study

\begin{tabular}{|c|l|l|l|l|}
\hline \multicolumn{1}{|c|}{} & \multirow{2}{*}{ Grid Size } & \multicolumn{3}{|c|}{ Kn } \\
\cline { 3 - 5 } & & $\mathbf{0 . 0 0}$ & $\mathbf{0 . 0 2}$ & $\mathbf{0 . 0 4}$ \\
\hline \multirow{3}{*}{$\begin{array}{c}\text { Current } \\
\text { Study }\end{array}$} & $16 \times 384$ & 7.58 & 6.98 & 6.42 \\
\cline { 2 - 5 } & $48 \times 768$ & 7.56 & 6.94 & 6.39 \\
\cline { 2 - 5 } & $80 \times 1536$ & 7.55 & 6.89 & 6.26 \\
\hline Celik[6] & $21 \times 420$ & 7.57 & 6.94 & 6.38 \\
\cline { 2 - 5 } & $41 \times 820$ & 7.56 & 6.94 & 6.38 \\
\cline { 2 - 5 } & $81 \times 1620$ & 7.55 & 6.93 & 6.37 \\
\hline
\end{tabular}




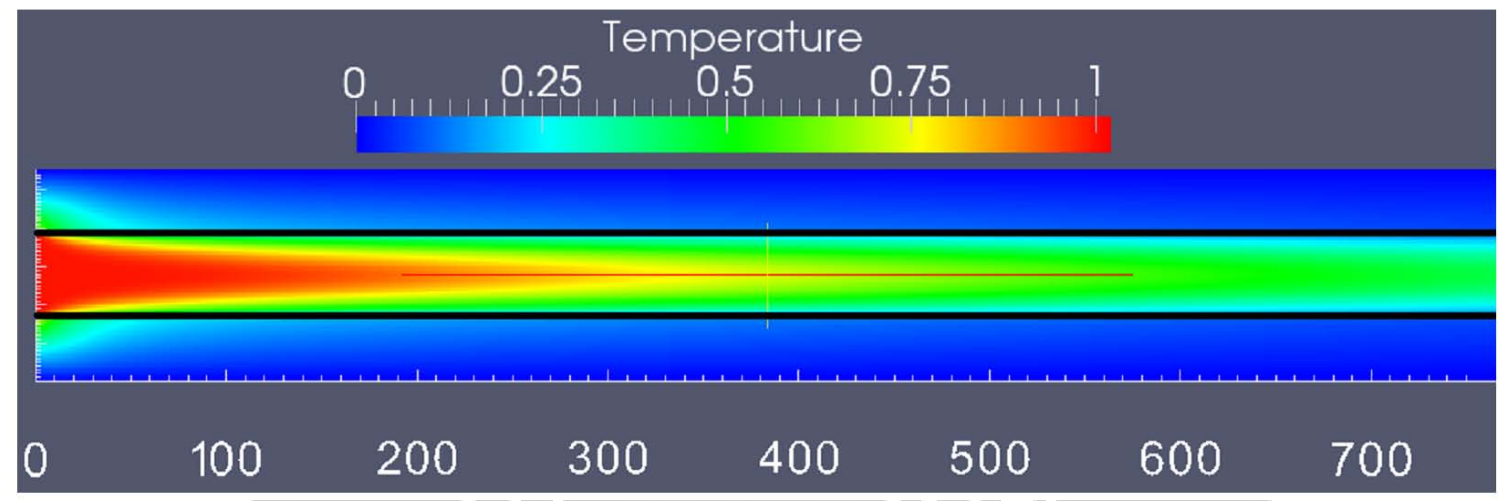

FIGURE 8. STEADY STATE ISOTHERM OF CASE 2 WITH CONJUGATE HEAT TRANSFER

The GPU performances are compared with CPU performance for $\mathrm{Kn}=0.02$ and $\mathrm{k}=1.667$ on the three types of grids. To obtain the steady state, all simulations were run for 60000 time steps. The GPU code performs $0.76,2.63$ and 15.12 times faster than CPU as shown in Table 2. The speedup numbers increases as the size of grids increases, because the intensity of arithmetic calculation increases with the grid sizes and the data communications in the memory of the GPU compared to the time spent on computation becomes shorter.

Table 2. Comparison of performance for GPU vs CPU

\begin{tabular}{|l|r|r|r|}
\hline Grids & $\mathbf{1 6 \times 3 8 4}$ & $\mathbf{4 8 \times 7 6 8}$ & $\mathbf{8 0 \times 1 5 3 6}$ \\
\hline CPU Execution Time (s) & 73.35 & 902.58 & 14907.90 \\
\hline GPU Execution Time (s) & 96.46 & 342.83 & 985.96 \\
\hline Speed up & 0.76 & 2.63 & 15.12 \\
\hline
\end{tabular}

\section{CASE 2. WITH CONJUGATE HEAT TRANSFER}

Grid size $112 \times 768$ was used in this case, the microchannel width was 47 units and the length was 767 . The microchannel was located in the middle of the domain as shown in figure 3 . The Knudsen number is 0.02 and $k=0$ (this number indicates that there is no jump temperature at the fluid-solid interface). The calculation results of the isotherms at steady state is shown in Figure 8. The heat conduction within the solid wall can be seen clearly in Figure 8 and 9. The isotherm of Case 2 as shown in Figure 8 shows the increase of the temperature of the wall along the edges of the inlet due to conjugate heat transfer maintains the higher temperature of the fluid longer compared to the Case 1 . The constant wall temperature present in Case 1 reduces the temperature of the fluid more rapidly than Case 2 .

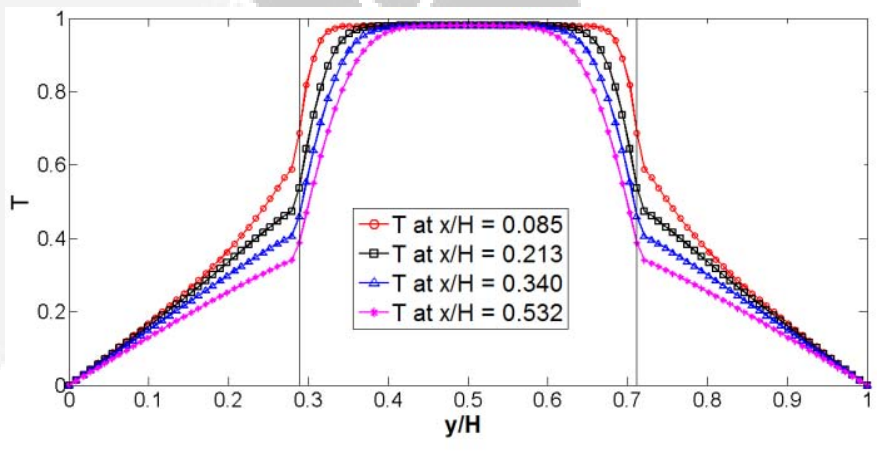

FIGURE 9. STEADY STATE TEMPERATURE PROFILES NEAR MICROCHANNEL INLET FOR CASE 2

Figure 9 describes the developing temperature profile near the inlet, both within the microchannel wall and within the fluid. As can be seen, the temperature of the wall increases signifcantly due to the heat transfer between the fluid and the wall. Comparing the temperature profiles to Case 1 temperature profile in Figure 4, it can be seen clearly that the temperature of the fluid when incorporating conjugate heat transfer is higher at the equivalent position within the microchannel. For example at $\mathrm{x} / \mathrm{H}=0.53$ the normalized temperature of the fluid at the wall for Case 1 is around 0.4 compared to slightly less than 0.2 for the equivalent position for Case 2. While the Case 2 model did not display temperature jump features as Case 1 did, the presence of temperature jump would further increase the temperature of the fluid of Case 2. This significant temperature different accentuates the importance of the consideration of conjugate heat transfer. 


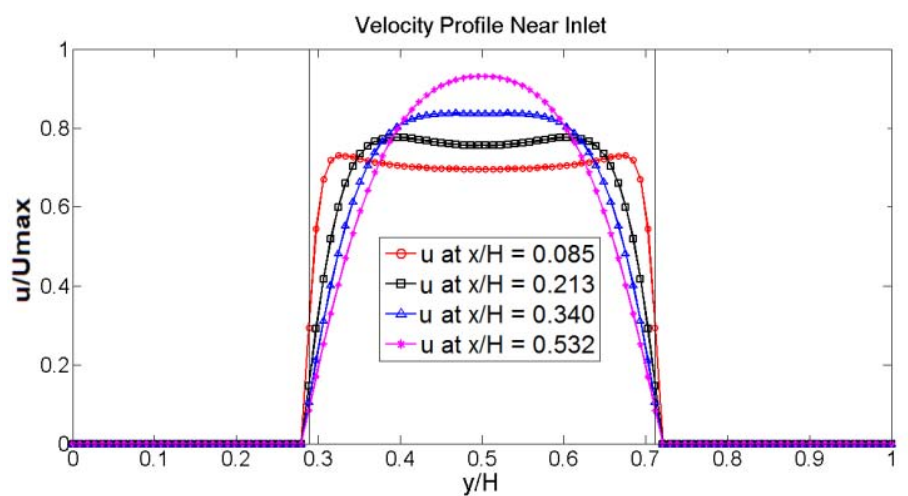

FIGURE 10. STEADY STATE VELOCITY PROFILES NEAR MICROCHANNEL INLET FOR CASE 2

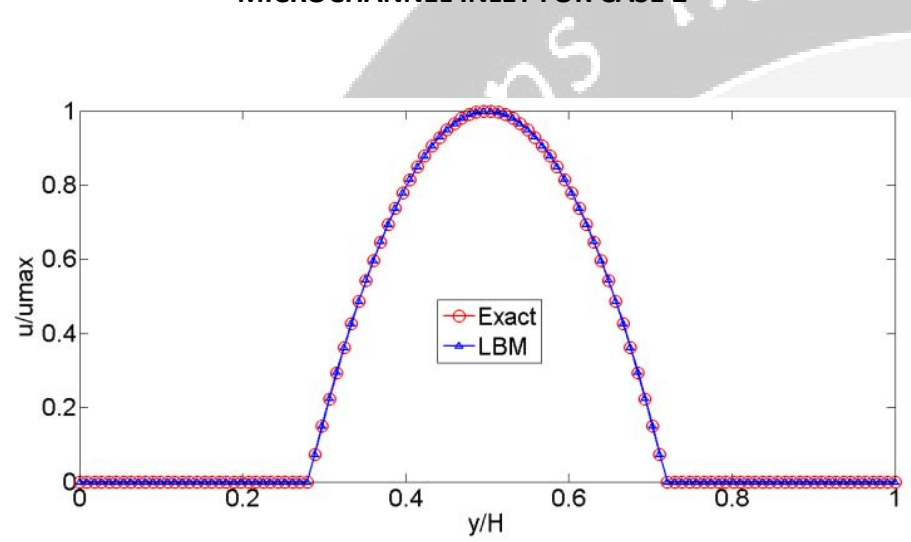

FIGURE 11. COMPARISON OF FULLY DEVELOPED VELOCITY PROFILES OF CASE 2 MODEL AND EXACT SOLUTION FOR X/H $=2.128$

Figure 10 shows the developing velocity profiles near the inlet. The difference between Case 1 and Case 2 in regards to velocity profile are insignificant. Therefore the comparison of the numerical results of velocity profile to the exact solution given by Equation 12[16] again show excellent agreement (Figure 11).

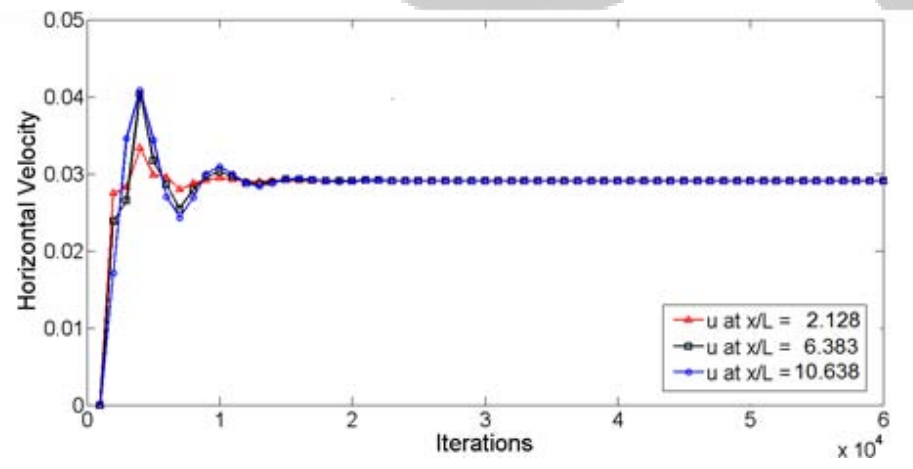

FIGURE 12. VELOCITY HISTORY

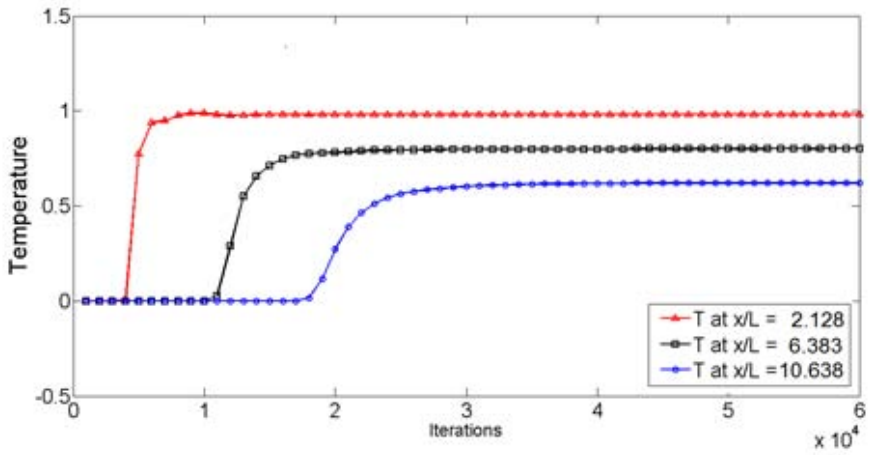

FIGURE 13. WALL SURFACE TEMPERATURE HISTORY

The transient results for velocity is shown in Figure 12. As can be seen, steady state hydrodynamics is obtained at around time step 15000 . From the transient wall surface temperature results shown in Figure 13, it can be seen that steady state temperature is achieved earlier at points closer to the inlet. As it can be observed that there is no visible change of wall temperature and velocity for more than 40000 time steps, one can concludes that at 60000 time steps, steady state has been achieved.

As it was for Case 1, for Case 2 with conjugate heat transfer, the use of the GPU was capable of accelerating computation times. Computation with a sole CPU required a computation time of 1999.98 seconds. The GPU reduced computation time to 650.95 second, thus increasing processing speed up to 3.07 times faster.

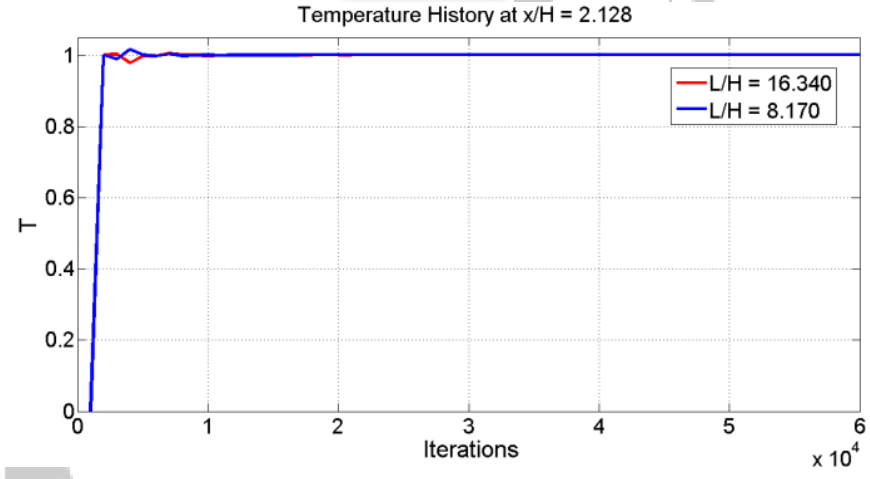

FIGURE 14. ASPECT RATIO INDEPENDENCY COMPARISON OF TEMPERATURE AT $\mathrm{X} / \mathrm{H}=\mathbf{2 . 1 2 8}$

To confirm the dependency of results on model aspect ratio, we have run additional trials comparing microchannel models with aspect ratios of $\mathrm{L} / \mathrm{H}=16.340$ and $\mathrm{L} / \mathrm{H}=8.170$. Figures 14 and 15 show temperature and velocity history at position $\mathrm{x} / \mathrm{H}=$ 2.218, while Figures 16 and 17 show the history at $\mathrm{x} / \mathrm{H}=6.383$. It can be seen that transient temperature results are fairly consistent between the two aspect ratios, as is velocity for $\mathrm{x} / \mathrm{H}=2.218$. However, at $\mathrm{x} / \mathrm{H}=6.383$ there is a difference between time steps 1000 to 5000 . Approaching steady state 
and after steady state is achieved, results for both aspect ratios in regards to temperature and velocity are the same.

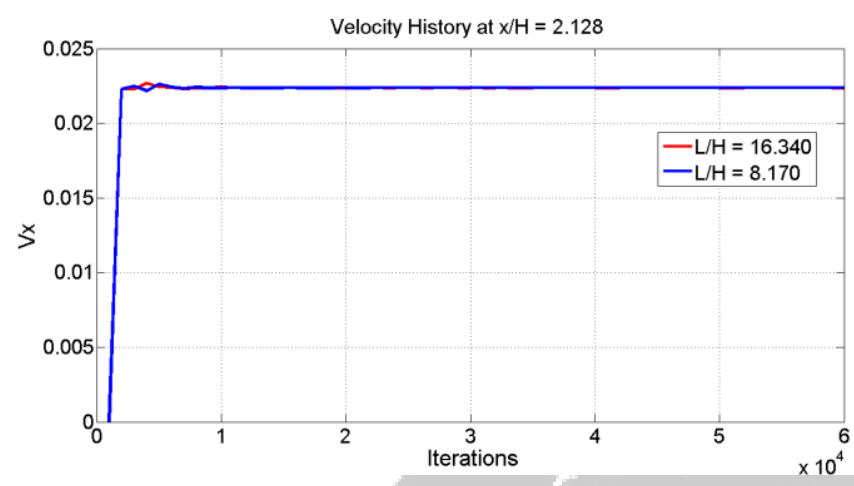

FIGURE 15. ASPECT RATIO INDEPENDENCY COMPARISON OF VELOCITY AT $X / H=2.128$

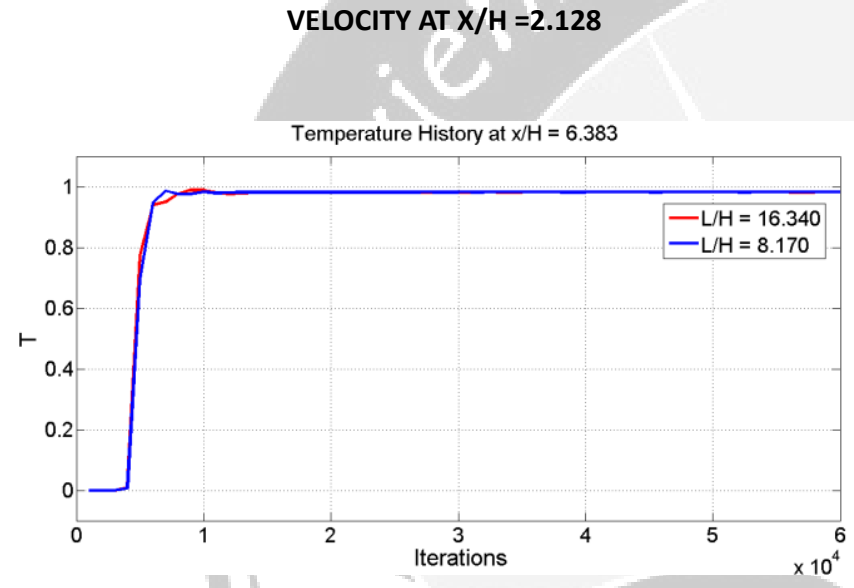

FIGURE 16. ASPECT RATIO INDEPENDENCY COMPARISON OF TEMPERATURE AT $\mathrm{X} / \mathrm{H}=6.383$

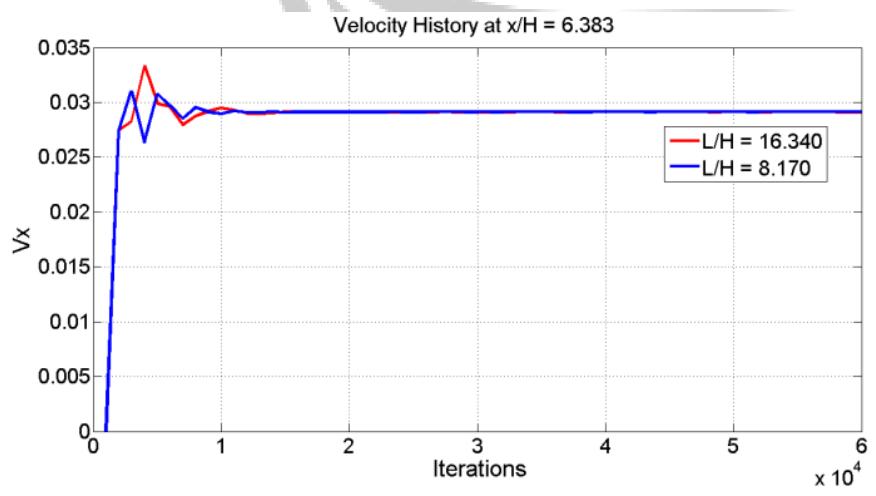

FIGURE 17. ASPECT RATIO INDEPENDENCY COMPARISON OF VELOCITY AT $\mathrm{X} / \mathrm{H}=6.383$

\section{CONCLUSION}

We have presented the Lattice Boltzmann method for simulation of conjugate convective-conductive heat transfer in a microchannel within the slip regime. Two cases have been considered : fluid flow and heat transfer without consideration of conjugate heat transfer (Case 1) and with consideration of conjugate heat transfer (Case 2).

Velocity profile results between Case 1 and Case 2 were similar as the effect of temperature to momentum were insignificant. We have validated the velocity profile numerical results to exact solution equations and achieved good agreement. Interestingly, temperature profile results have shown that consideration of conjugate heat transfer within the simulation reveals fluid temperatures at the microchannel wall to be significantly higher than consideration of constant wall temperature. This fact highlights the importance of the consideration of conjugate heat transfer in microflow modeling.

To evaluate the dependency of the model on aspect ratio, we have compared models with two different aspect ratios : $\mathrm{L} / \mathrm{H}=8.170$ and $\mathrm{L} / \mathrm{H}=16.340$. Results have shown that steady state results are not influenced by differences in aspect ratio. However, care must be taken when evaluating transient results since a difference of velocity calculation between the two aspect ratios was obtained at early time steps. This difference may be due to numerical effects when determining boundary conditions of the exit. Further work will be conducted to improve the transient computation.

To reduce computation times, our model incorporated GPU parallel processing. Comparison of calculation times has shown that this has increased processing speed up from 0.76 to 15.12 faster than lone CPU computation depending on the size of the grids.

\section{REFERENCES}

[1] Kandlikar, S. G., 2006, Heat transfer and fluid flow in minichannels and microchannels, Elsevier Science Limited.

[2] Sung, M. K., and Mudawar, I., 2006, "Experimental and numerical investigation of single-phase heat transfer using a hybrid jet-impingement/micro-channel cooling scheme," International journal of heat and mass transfer, 49(3), pp. 682694.

[3] Li, Z., He, Y. L., Tang, G. H., and Tao, W. Q., 2007, "Experimental and numerical studies of liquid flow and heat transfer in microtubes," International journal of heat and mass transfer, 50(17), pp. 3447-3460.

[4] Shu, C., Niu, X., and Chew, Y., 2005, "A lattice Boltzmann kinetic model for microflow and heat transfer," Journal of statistical physics, 121(1), pp. 239-255.

[5] Ansumali, S., Karlin, I., Frouzakis, C. E., and Boulouchos, K., 2006, "Entropic lattice Boltzmann method for microflows," 
Physica A: Statistical Mechanics and its Applications, 359, pp. 289-305.

[6] Celik, S. B., 2012, "Analysis of Single Phase Fluid Flow and Heat Transfer in Slip Flow Regime by Parallel Implementation of Lattice Boltzmann Method on GPUs," M.Sc. Dissertation, Middle East Technical University, Ankara, Turkey.

[7] Rosa, P., Karayiannis, T., and Collins, M., 2009, "Single-phase heat transfer in microchannels: The importance of scaling effects," Applied Thermal Engineering, 29(17), pp. 3447-3468.

[8] Mohammadi Pirouz, M., Farhadi, M., Sedighi, K., Nemati, H., and Fattahi, E., 2011, "Lattice Boltzmann simulation of conjugate heat transfer in a rectangular channel with wallmounted obstacles," Scientia Iranica, 18(2), pp. 213-221.

[9] Wang, J., Wang, M., and Li, Z., 2007, "A lattice Boltzmann algorithm for fluid-solid conjugate heat transfer," International journal of thermal sciences, 46(3), pp. 228-234.

[10] Sukop, M., and Thorne, D., 2006, "Lattice Boltzmann Modeling: An Introduction for Geoscientists and Engineers.," Springer-Verlag, Netherlands.

[11] Tian, Z. W., Chun, Z., Liu, Z. H., Guo, Z. L., Hong-Juan, L., and Zheng, C. G., 2006, "Lattice Boltzmann method in simulation of thermal micro-flow with temperature jump," International Journal of Modern Physics C, 17(05), pp. 603-614.

[12] Thibault, J. C., and Senocak, I., "CUDA Implementation of a Navier-Stokes Solver on Multi-GPU Desktop Platforms for Incompressible Flows," Proc. 47th AIAA Aerospace Sciences Meeting.

[13] Tölke, J., 2010, "Implementation of a Lattice Boltzmann kernel using the Compute Unified Device Architecture developed by nVIDIA," Computing and Visualization in Science, 13(1), pp. 29-39.

[14] Sanders, J., and Kandrot, E., 2011, "CUDA by Example," An Introduction to General-Purpose GPU Programming, 1st ed. Upper Saddle River, NJ: Addison-Wesley Professional.

[15] Pullan, G., 2013, "A 2D Lattice Boltzmann Flow Solver Demo,"http://www.many-core.group.cam.ac.uk/projects /LBdemo.shtml"

[16] Karniadakis, G., Beskok, A., and Aluru, N., 2005, Microflows and nanoflows: fundamentals and simulation, Springer. 